\title{
Functionalized viral nanoparticles as ultrasensitive reporters in lateral-flow assays $\dagger$
}

Cite this: Analyst, 2013, 138, 5584

Received 1st May 2013

Accepted 23rd July 2013

DOI: 10.1039/c3an00891f

www.rsc.org/analyst

Two types of viral nanoparticles were functionalized with targetspecific antibodies and multiple copies of an enzymatic reporter (horseradish peroxidase). The particles were successfully integrated into an immunochromatographic assay detecting MS2 bacteriophage, a model for viral pathogens. The sensitivity of the assay was greatly superior to conventional gold nanoparticle lateral flow assays, and results could easily be evaluated, even without advanced lab instruments.

Immunochromatographic lateral flow assays (LFAs) are a wellestablished, inexpensive point-of-need diagnostic analytical method with proven utility for the primary testing of diverse samples. LFAs quickly provide a qualitative or semi-quantitative result without the need for elaborate sample preparation or capital investment. In conventional LFAs colloidal gold, selenium, blue latex or carbon nanoparticles bearing antibodies to the target are used as the reporter, which is analyte-bridged to a capture antibody attached to the surface of a nitrocellulose strip. The appearance of a line of captured reporter particles indicates a positive result, whilst a control line further downstream demonstrates that the test has performed correctly. LFAs have been used to detect a wide range of biomolecules, including nucleic acids, proteins, toxins, and hormones, as well as bacterial and viral pathogens. ${ }^{1}$ However, the sensitivity of LFAs, particularly for detecting viruses, lags behind more elaborate laboratory methods, such as plaque counting or PCR. ${ }^{2,3}$ For example, colloidal gold LFA detection of Japanese encephalitis virus in swine are reported to require at

${ }^{a}$ Department of Biology and Biochemistry, University of Houston, Houston, TX 77204-5001, USA

${ }^{b}$ Department of Chemical and Biomolecular Engineering, University of Houston, Houston, TX 77204-4004, USA

'Houston Methodist Research Institute, Houston, TX 77030, USA. E-mail: willson@uh. edu; Tel: +1 713-743-4308

$\dagger$ Electronic supplementary information (ESI) available. See DOI: 10.1039/c3an00891f

$\ddagger$ These authors contributed equally to this work. least $2.5 \times 10^{6} \mathrm{pfu} \mathrm{mL}^{-1} \cdot{ }^{4} \mathrm{~A}$ comparable assay for filamentous Escherichia coli M13 phage was able to detect $5 \times 10^{7} \mathrm{pfu} \mathrm{mL}^{-1} .^{5}$

Functionalized viral nanoparticles have previously been reported to be suitable as affinity reagents in enzyme-linked immunosorbent assays (ELISAs). ${ }^{6-8}$ Here, we present two different approaches to the manufacture of antibody- and peroxidase-doubly-modified M13 bacteriophage, and their use in LFAs (Fig. 1). In the first approach, horseradish peroxidase (HRP) and target specific antibodies are chemically attached to the abundant coat protein (pVIII) of M13. In an alternative

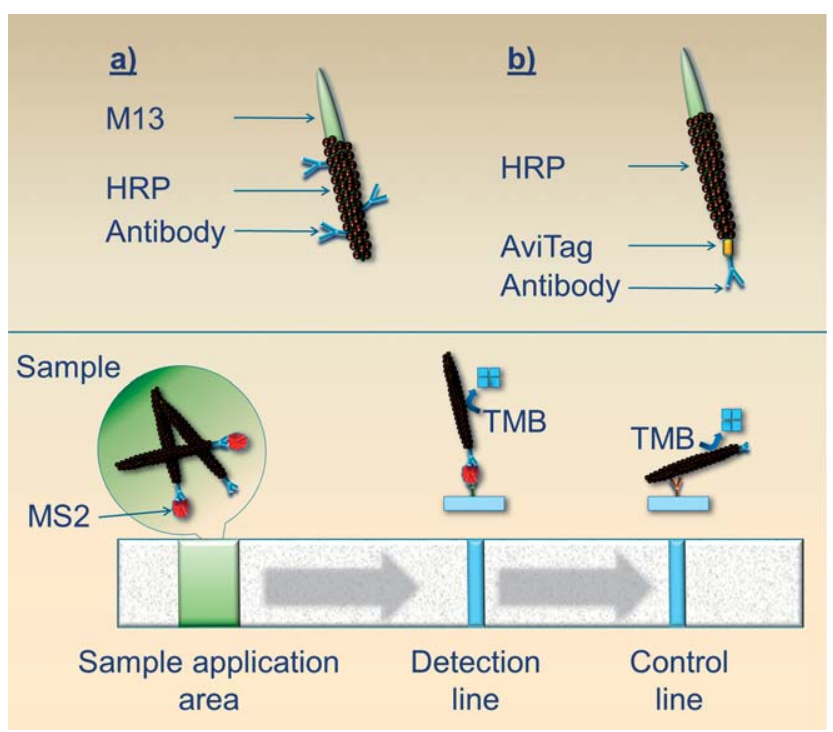

Fig. 1 Viral nanoparticles as lateral-flow reporters. (Top panel) two types of nanoparticles were used. (a) The M13 coat proteins were functionalized with horseradish peroxidase (HRP) and analyte-specific antibodies. (b) The SAM-AviTag protein, plll, was specifically biotinylated and functionalized with analyte-specific antibodies, and the coat proteins were functionalized with HRP. (Bottom panel) lateral flow assay for MS2 bacteriophage. The detection line contains MS2-specific antibodies, and the control line has anti-M13 antibodies. HRP-labelled phage were detected using the chromogenic substrate, 3,3',5,5'-tetramethylbenzidine (TMB). 
approach, SAM-AviTag phage, derivatives of phage M13 where the N-terminus of the few-copy phage coat protein III contains the enzymatically biotinylatable AviTag peptide (GLNDIFEAQKIEWHE), are employed. The lysine residue (K) in the AviTag is a substrate for biotinylation by $E$. coli biotin ligase (birA). Using streptavidin or neutravidin, any biotinylated affinity agent can then easily be linked to these enzymatically biotinylated phage particles. ${ }^{9-11}$ Notably, both approaches are suitable for the attachment of intact antibodies, (rather than scFvs, which require cloning and which often have lower affinity than the parent antibody).

The use of gold nanoparticles in combination with the enzymatic activity of horseradish peroxidase (HRP) has been shown to improve the sensitivity of LFAs by one order of magnitude. ${ }^{12}$ LFAs for $E$. coli bacteriophage MS2 have previously been commercialized as a tool for the detection of $E$. coli (MicroPhage Inc., Golden, CO) after phage expansion, but no limit of detection has been published.

\section{Methods}

\section{Culture and titration of MS2 bacteriophage}

Bacteriophage MS2 (\#15597-B1) and its E. coli host strain (\#15597) were obtained from the American Type Culture Collection (Manassas, VA). For MS2 propagation, the E. coli host was propagated in tryptic soy broth (TSB) at $37{ }^{\circ} \mathrm{C}$ with shaking until log phase. For phage enrichment, $75 \mu \mathrm{L}$ of bacterial host culture and $100 \mu \mathrm{L}$ of MS2 stock $\left(\sim 10^{9} \mathrm{pfu} \mathrm{mL}^{-1}\right)$ were added to a sterile tube containing $5 \mathrm{~mL} 0.7 \%$ tryptic soy agar (TSA), kept at $48{ }^{\circ} \mathrm{C}$. The contents of the tube were gently mixed, and then carefully poured onto a pre-warmed $1.5 \%$ TSA plate. After overnight incubation at $37{ }^{\circ} \mathrm{C}$, phage were harvested by gently scraping the top layer of soft agar into a $50 \mathrm{~mL}$ tube. Equal volumes $(\sim 20 \mathrm{~mL})$ PBS and chloroform were added and the mixture was vortexed for $5 \mathrm{~min}$. After $30 \mathrm{~min}$ centrifugation at $4000 \times g$ the MS2-containing aqueous phase was carefully removed and filtered through sequentially smaller low protein-binding filters $(0.45 / 0.22 \mu \mathrm{m})$. Aliquots of the MS2 stocks were stored at $-80{ }^{\circ} \mathrm{C}$. For phage titering, dilutions of the phage stock were mixed with the E. coli host and the TSA, and poured onto 1.5\% TSA plates. Individual plaques were counted after overnight incubation. ${ }^{13}$

\section{Culture and titration of M13 bacteriophage}

SAM-Avitag M13 phage were a gift from Dr Brian Kay, UIC (Chicago, IL). For phage enumeration, E. coli TG1 was grown to mid-log phase in LB at $37{ }^{\circ} \mathrm{C}$ with shaking. The bacterial culture ( $5 \mathrm{~mL})$ was then infected with $5 \mu \mathrm{L}$ of phage stock $\left(\sim 10^{12} \mathrm{pfu}\right.$ $\mathrm{mL}^{-1}$ ) and grown for $2 \mathrm{~h}$ at $37^{\circ} \mathrm{C}$ with shaking. This pre-culture was then transferred to $500 \mathrm{~mL} 2 \times$ TY medium and incubated overnight at $37{ }^{\circ} \mathrm{C}$ on a shaker. Bacteria were separated from phage in the supernatant by centrifugation $(30 \mathrm{~min}, 3200 \times g)$ and filtration through $0.45 \mu \mathrm{m}$ filters. Phage were then precipitated using PEG/NaCl as previously described. ${ }^{\mathbf{1 4}}$ The concentrated phage were stored at $4{ }^{\circ} \mathrm{C}$ with $0.02 \%$ sodium azide. Phage titers were determined on X-Gal/IPTG plates as described. ${ }^{15}$

\section{Bacteriophage M13 functionalization through avidin-biotin}

SAM-AviTag phage were enzymatically biotinylated using E. coli biotin ligase (Avidity, Aurora, CO, or prepared in-house) according to the manufacturer's instructions. The biotinylated phage were then covalently coupled to horseradish peroxidase (HRP, Sigma-Aldrich, St. Louis, MO) using Traut's reagent (2iminothiolane-HCl, Thermo Fisher Scientific, Waltham, MA). Briefly, $100 \mu \mathrm{L}\left(\sim 10^{11}\right)$ phage were suspended in $800 \mu \mathrm{L}$ PBS, 3 mM EDTA. Traut's reagent was added to a final concentration of $7 \mu \mathrm{M}$ and the reaction was allowed to continue for $90 \mathrm{~min}$ at $25{ }^{\circ} \mathrm{C}$. Excess Traut's reagent was removed using $10 \mathrm{kDa}$ filters (Cat. no. UFC901024, Millipore, Billerica, MA). Maleimide-HRP was separately prepared by mixing HRP and sulfo-SMCC (succinimidyl-4-( $N$-maleimidomethyl)cyclohexane-1-carboxylate; Thermo Fisher Scientific) for $30 \mathrm{~min}$ to achieve final concentrations of $22 \mu \mathrm{M}$ and $1.14 \mathrm{mM}$, respectively, in $1 \mathrm{~mL}$ PBS. Excess sulfo-SMCC was removed using a $10 \mathrm{kDa}$ filter. Traut's reagent-modified phage and sulfo-SMCC-modified HRP were then mixed for $90 \mathrm{~min}$ at $25{ }^{\circ} \mathrm{C}$. Free HRP was removed using $100 \mathrm{kDa}$ filters (Millipore). Neutravidin (Thermo Fisher Scientific) was bound to the HRP-labelled phage through the biotinylated AviTag displayed on phage protein III. Rabbit-AntiMS2 polyclonal antibody was purchased from Tetracore, Inc. (Gaithersberg, MD), and biotinylated using EZ-Link Sulfo-NHSLC-Biotin (Thermo Fisher Scientific) reagent using a 20 -fold molar excess of biotin reagent according to the manufacturer's instructions. Neutravidin-functionalized phage and biotinylated antibody (at a 10-fold molar excess) were incubated for $1 \mathrm{~h}$ at $37^{\circ} \mathrm{C}$, before uncoupled antibodies were removed using a $300 \mathrm{kDa}$ spin dialyzer (Harvard Apparatus, Holliston, MA).

\section{Bacteriophage M13 functionalization through covalent coupling of antibody and reporter enzyme}

In this approach, M13 phage were activated using Traut's reagent as described above. Maleimide-HRP and maleimide anti-MS2 antibodies were separately prepared as described above for HRP activation, and combined with Traut's reagent-activated M13 phage particles at a molar ratio (HRP : Antibody: Phage) of $270000: 10: 1$. After $90 \mathrm{~min}$ at $25{ }^{\circ} \mathrm{C}$, the complex was dialyzed using a $300 \mathrm{kDa}$ spin dialyzer (Harvard Apparatus) to remove free enzyme and antibody.

\section{Preparation of LFA strips}

To form MS2-detection and M13 control lines, $5 \mu \mathrm{L}$ anti-MS2 antibodies $\left(0.4 \mu \mathrm{g} \mu \mathrm{L}^{-1}\right)$ and $5 \mu \mathrm{L}$ anti-M13 antibodies (0.1 $\mu \mathrm{g} \mu \mathrm{L}^{-1}$ ), (GE Healthcare, Piscataway, NJ), both in $50 \mathrm{mM}$ sodium acetate buffer ( $\mathrm{pH}$ 3.6), were spotted on Fusion 5 membranes (GE Healthcare Biosciences, Piscataway, NJ) using a Lateral Flow Reagent Dispenser (Claremont BioSolutions, Upland, CA) equipped with an external syringe pump (Chemyx, Stafford, TX). The strips were allowed to dry for $1 \mathrm{~h}$ at $25{ }^{\circ} \mathrm{C}$. We also explored the use of nitrocellulose, commonly used in conventional immunochromatographic assays, with similar results as for Fusion 5, though a noticeably higher background was observed. 


\section{Lateral flow assay}

The bacteriophage MS2 test analyte was diluted in LFA buffer (100 mM Tris-HCl, pH 8; 0.3\% Tween-20; 0.2\% BSA; 0.1\% PEG3350). $100 \mu \mathrm{L}$ samples were pipetted onto the sample application area of a prepared $7 \times 50 \mathrm{~mm}$ Fusion 5 strip with anti-MS2 antibodies on the detection line and anti-M13 antibodies on the control line. The strips were first washed with $200 \mu \mathrm{L}$ test buffer before $10 \mu \mathrm{L}$ of the respective phage construct $\left(\sim 10^{8}\right)$ were dispensed onto the distal end of the LFA strip. The strips were washed again with $500 \mu \mathrm{L}$ LFA buffer, before signals were obtained by spotting $25 \mu \mathrm{L}$ TMB Liquid Substrate System for Membranes (Sigma-Aldrich) on each line. The strips were scanned in a Perfection V600 flatbed color scanner (Epson, Long Beach, CA).

\section{Results and discussion}

While immunochromatographic assays are a widely useful method for point-of-care diagnostics, there is a felt need for improved LFA analytical sensitivity. This can be addressed by pre-concentrating the target analyte, ${ }^{5,16}$ or, as described in this work, by developing improved reporter particles.

In order to obtain a stronger signal per individual affinity agent, we employed viral nanoparticles as the scaffold to which we chemically attached multiple reporter enzymes. Even considering mutual steric exclusion, the 2700 pVIII coat proteins of phage M13 likely offer hundreds of binding sites for reporters such as horseradish peroxidase, as well as for antibodies specific for the target of interest. For the addition of antibodies to these phage affinity agents, we compared two approaches, the covalent linkage of the antibodies to the phage coat proteins and the avidin-mediated coupling of biotinylated antibodies to enzymatically biotinylated AviTag peptides displayed on the phage tail proteins.

The model analyte used in our study, the E. coli bacteriophage MS2, has been widely used as a model for viral pathogens, and is used as an indicator of water quality by the United States Environmental Protection Agency. Serial dilutions of the phage were prepared from a stock whose titer had been determined using the double agar overlay plaque assay, the standard for the enumeration of $\mathrm{MS}^{2,{ }^{13}}$ and independently confirmed spectrophotometrically. ${ }^{17}$ Anti-MS2 antibodies were spotted at the detection line to capture any MS2 phage and subsequently any viral nanoparticles with anti-MS2 antibodies (Fig. 2A and B). After adding the enzyme substrate, the LFA strips were scanned and the data were evaluated using Image $\mathrm{J}^{\prime}$ ' density analysis function ${ }^{\mathbf{1 8}}$ (Fig. 2C). A signal was obtained at the anti-M13 control line for all samples, indicating that the viral nanoparticle reporters successfully moved through the membrane. At the test line, the signal obtained for $10^{3}$ pfu of MS2 was clearly distinguishable from the control containing no phage (Fig. 2D). Given a sample volume of $100 \mu \mathrm{L}$, this corresponds to a titer of $10^{4} \mathrm{pfu} \mathrm{mL}^{-1}$, which represents almost one thousand-fold increased sensitivity compared to gold nanoparticle LFA with the same antibodies (see ESI $\dagger$ ), and at least a one hundred-fold improvement in sensitivity over

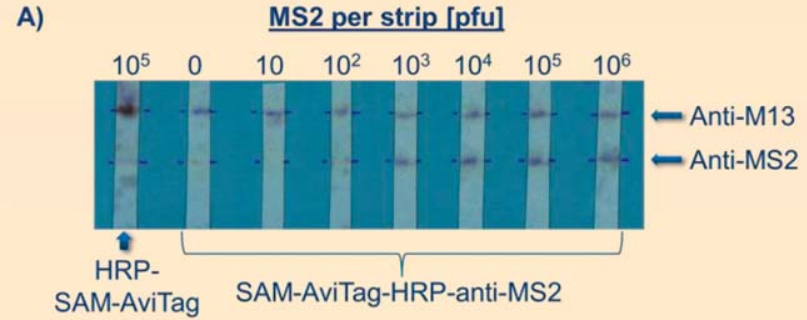

B)

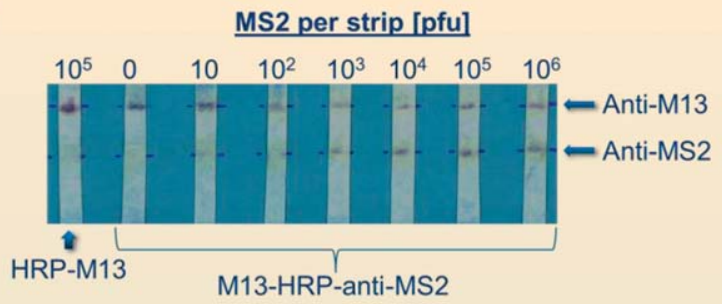

C)

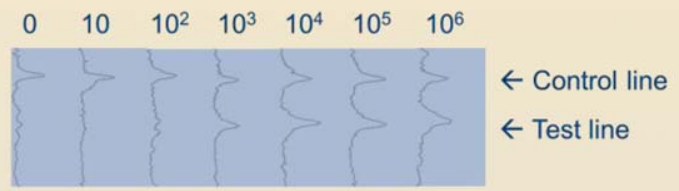

D)

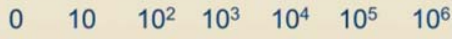

$$
\begin{aligned}
& \leftarrow \text { Control line } \\
& \leftarrow \text { Test line }
\end{aligned}
$$

E)

\begin{tabular}{|c|c|c|}
\hline \multicolumn{3}{|c|}{ Relative Intensity } \\
\hline Phage/Strip & Test Line (A) & Test Line (B) \\
\hline 0 & 0 & 0 \\
\hline 10 & 0 & 0 \\
\hline 100 & 318 & 0 \\
\hline 1,000 & 3235 & 2100 \\
\hline 10,000 & 6387 & 3555 \\
\hline 100,000 & 5313 & 4662 \\
\hline $1,000,000$ & 7486 & 3856 \\
\hline \multicolumn{3}{|c}{} \\
\end{tabular}

Fig. 2 Immunochromatographic detection of bacteriophage MS2. (A) Various numbers of MS2 phage were added to a Fusion 5 strip containing anti-MS2 and anti-M13 antibodies. Anti-MS2 antibodies were added to a biotin on the tail protein (pIII) of the M13 derivative, SAM-AviTag via avidin-biotin conjugation. Horseradish peroxidase (HRP) was chemically linked to the coat proteins, pVIII. SAM-AviTag phage carrying just the HRP reporter (HRP-M13) enzyme were used as a control. (B) Here M13 phage, chemically functionalized with anti-MS2 antibodies and horseradish peroxidase were added to bind to both the detection (anti-MS2; in the presence of analyte) and control (anti-M13) lines. M13 phage carrying just the HRP reporter enzyme (HRP-M13) were used as a control (leftmost strip). (C) Image J density analysis of the LFA strips in (A). (D) Image J density analysis of the LFA strips in (B). (E) Relative intensities of the test lines shown in (A) and $(B)$, as determined by Image $J$.

previously reported LFAs for viral detection. ${ }^{\mathbf{4}, 5}$ In addition, we have shown that without anti-MS2 antibodies the M13 phage is not non-specifically retained by the anti-MS2 antibodies. Data for three independent repeat experiments for each type of viral nanoparticle is presented in the ESI. $\dagger$ It shows identical limits of detection.

We did not observe any significant difference in sensitivity between M13 phage containing anti-MS2 antibodies attached to the pIII tail protein, and those with the antibodies attached to 
the phage coat. Assuming that the number of pIII molecules is between three and five per phage, and the number of pVIII coat proteins is 2700 , we would have expected a significantly larger number of antibodies per phage in the latter approach.

The success of M13 reporters with low antibody loadings is consistent with the successful practice of phage-display library screening in the "monovalent phage display" format, in which a helper phage technique is used to display only a single genetically encoded candidate antibody on the M13 tail protein, eliminating the confounding effects of multivalent binding by multiple copies of the displayed antibody. ${ }^{19,20}$ The success of this method implies that phage are retained at significant efficiency by affinity of a single displayed antibody, and argues that M13 LFA reporters should display small numbers of antibodies and a maximal number of enzyme reporters.

We are actively pursuing the optimization of the assay, including its integration into a paper microfluidics system that will reduce the number of hands-on steps. We are also investigating the exact effect of varying the number of functionalized viral nanoparticles used in the assay and the number of enzymes and antibodies per viral particle. We are further optimistic that the use of reporter enzymes other than HRP (with or without the combination with chemiluminescent substrates) will yield higher sensitivity. Moreover, alternative membranes to optimize the assay will be tested, though with nitrocellulose, while essentially yielding the same level of detection as Fusion 5 , we did observe a higher background signal with our phage. Lastly, we are currently investigating functionalized viral nanoparticles with different morphologies as alternative reporters.

\section{Conclusions}

We have found that M13 phage decorated with peroxidase enzymes and anti-analyte antibodies can serve as reporters in lateral-flow assays of greatly enhanced sensitivity. In the future, we will examine the use of alternative enzyme reporters and substrates for our LFA scheme, especially chemiluminescent substrates, and extend the use of this approach to detection of pathogenic viruses in blood serum.

\section{Acknowledgements}

Postdoctoral scholarships to A.E.V. Hagström from the Olle Engkvist Byggmästare foundation and the Carl Trygger foundation are gratefully acknowledged.

\section{Notes and references}

1 G. A. Posthuma-Trumpie, J. Korf and A. van Amerongen, Anal. Bioanal. Chem., 2009, 393, 569-582.

2 T. M. Uyeki, R. Prasad, C. Vukotich, S. Stebbins, C. R. Rinaldo, Y. H. Ferng, S. S. Morse, E. L. Larson, A. E. Aiello, B. Davis and A. S. Monto, Clin. Infect. Dis., 2009, 48, e89-92.

3 M. M. Izzo, P. D. Kirkland, X. Gu, Y. Lele, A. A. Gunn and J. K. House, Aust. Vet. J., 2012, 90, 122-129.

4 Y. Li, L. Hou, J. Ye, X. Liu, H. Dan, M. Jin, H. Chen and S. Cao, J. Virol. Methods, 2010, 168, 51-56.

5 F. Mashayekhi, R. Y. Chiu, A. M. Le, F. C. Chao, B. M. Wu and D. T. Kamei, Anal. Bioanal. Chem., 2010, 398, 29552961.

6 H. J. Kim, M. A. Rossotti, K. C. Ahn, G. G. Gonzalez-Sapienza, S. J. Gee, R. Musker and B. D. Hammock, Anal. Biochem., 2010, 401, 38-46.

7 H. J. Kim, K. C. Ahn, A. Gonzalez-Techera, G. G. GonzalezSapienza, S. J. Gee and B. D. Hammock, Anal. Biochem., 2009, 386, 45-52.

8 H. J. Kim, M. McCoy, S. J. Gee, G. G. Gonzalez-Sapienza and B. D. Hammock, Anal. Chem., 2011, 83, 246-253.

9 M. D. Scholle, F. R. Collart and B. K. Kay, Protein Expression Purif., 2004, 37, 243-252.

10 M. D. Scholle, J. W. Kehoe and B. K. Kay, Comb. Chem. High Throughput Screening, 2005, 8, 545-551.

11 M. D. Scholle, U. Kriplani, A. Pabon, K. Sishtla, M. J. Glucksman and B. K. Kay, ChemBioChem, 2006, 7, 834-838.

12 C. Parolo, A. de la Escosura-Muniz and A. Merkoci, Biosens. Bioelectron., 2013, 40, 412-416.

13 A. M. Kropinski, A. Mazzocco, T. E. Waddell, E. Lingohr and R. P. Johnson, Methods Mol. Biol., 2009, 501, 69-76.

14 S. Branston, E. Stanley, E. Keshavarz-Moore and J. Ward, Biotechnol. Prog., 2012, 28, 129-136.

15 T. Maniatis, E. F. Fritsch and J. Sambrook, Molecular cloning: a laboratory manual, Cold Spring Harbor Laboratory, Cold Spring Harbor, N.Y., 1982.

16 M. A. Nash, J. N. Waitumbi, A. S. Hoffman, P. Yager and P. S. Stayton, ACS Nano, 2012, 6, 6776-6785.

17 R. L. Stavis and J. T. August, Annu. Rev. Biochem., 1970, 39, 527-560.

18 C. A. Schneider, W. S. Rasband and K. W. Eliceiri, Nat. Methods, 2012, 9, 671-675.

19 H. B. Lowman, S. H. Bass, N. Simpson and J. A. Wells, Biochemistry, 1991, 30, 10832-10838.

20 H. B. Lowman and J. A. Wells, J. Mol. Biol., 1993, 234, 564578. 